\title{
Towards a macroscopically consistent discrete method for granular materials: Delaunay strain-based formulation
}

\author{
Göran Frenning ${ }^{1}$ (D) \\ Received: 30 August 2021 / Revised: 1 December 2021 / Accepted: 2 December 2021 / Published online: 24 December 2021 \\ (c) The Author(s) 2021
}

\begin{abstract}
We demonstrate that the Delaunay-based strain definition proposed by Bagi (Mech Mater 22:165-177, 1996) for granular media can be straightforwardly translated into a particle-based numerical method for continua. This method has a number of attractive features, including linear completeness and satisfaction of the patch test, exact conservation of linear and angular momenta in the absence of external forces and torques, and anti-symmetry of the gradient vectors for any two points not both on the boundary of the computational domain. The formulation in effect relies on nodal (particle) interpolation of the deformation gradient and is therefore inherently unstable. Drawing on the analogy with granular media, a pairwise interaction between particles is included to alleviate this issue. The underlying idea is to define a local, non-affine deformation of each bond or contact, and to introduce pairwise forces via a stored-energy functional expressed in terms of the corresponding local displacements. In this manner, a generalisation of the Ganzenmüller (Comput Methods Appl Mech Eng 286:87-106, 2015) hourglass stabilisation procedure to non-central forces is obtained. The performance of the method is demonstrated in a range of problems. This work can be considered a first step towards the development of a macroscopically consistent discrete method for granular materials.
\end{abstract}

Keywords Particle-based methods · Granular mechanics $\cdot$ Smoothed particle hydrodynamics $\cdot$ Discrete element method . Stabilisation

\section{Introduction}

Smoothed particle hydrodynamics (SPH) is a meshfree particle-based numerical method that was originally proposed by Lucy [36] and Gingold and Monaghan [21] in a cosmological context more than 40 years ago. The heart of the method is a kernel density estimate, i.e. the density of the material at a certain point is calculated from the number, mass and proximity of nearby particles using a smoothing kernel [34]. The method has a number of attractive features such as the ability to straightforwardly incorporate advection and convection, to naturally trace moving boundaries, to handle large deformation and its comparative ease of implementation [34]. Since its inception, SPH has been applied in a number of fields, including solid mechanics, starting with the work by Libersky and Petschek almost 30 years ago [31,32].

Göran Frenning

goran.frenning@farmbio.uu.se

1 Department of Pharmaceutical Biosciences and the Swedish Drug Delivery Center (SweDeliver), Uppsala University, Box 591, 75124 Uppsala, Sweden
However, it is well-known that the standard SPH formulation suffers from a number of issues that primarily are related to the stability of the method and the consistency of its underlying kernel and particle approximations. Firstly, the tensile instability, that causes particle clumping, as first observed by Swegle [52]. Secondly, spurious zeroenergy modes caused by rank deficiency of the stiffness matrix resulting from the commonly used particle (nodal) integration $[13,14]$. Thirdly, lack of linear completeness of the particle approximation for arbitrarily located particles. This issue is aggravated at boundaries, since the supporting domain of the kernel density estimate will be truncated at boundaries [35]. Fourthly, high-frequency pressure oscillations in shock-dominated problems, as a result of the collocation of mass at the particles [39,40]. These oscillations are particularly evident in the near-compressibility limit.

In the light of the Lax-Richtmyer equivalence theorem, stating that stability and consistency together are equivalent to convergence, these issues would appear to severely limit the practical usefulness of SPH. Fortunately, this is not so, because, there are a number of more or less efficient 
methods that can be used to resolve them. Whereas spurious zero-energy modes are a generic feature of SPH, the tensile instability is only present when Eulerian kernels are used [2]. It is thus possible to avoid the tensile instability using total $[43,56]$ or updated [54] Lagrangian formulations. It has also been shown that an artificial stress can counteract the tensile instability for Eulerian kernels, and the resulting algorithm, due to Grey et al. [24], has found widespread use. Drawing on the similarity with the hourglass modes commonly encountered in underintegrated finite elements, Ganzenmuller et al. have devised a promising means to alleviate the spurious zero-energy modes in SPH [16,18]. Procedures to restore consistency of the SPH approximations have been discussed in [35]. An often used correction was proposed in [5], which also addressed variational aspects of the SPH in order to derive formulations that preserve linear and angular momentum exactly in the absence of external forces and torques [4]. The first-order hyperbolic framework for large strain computational solid dynamics proposed by Bonet and co-workers $[3,20]$ is also a noteworthy line of developments. This formulation utilises an extended set of strain measures and results in a mixed form that has been implemented using an SPH approach with very good results in the near and truly incompressible limit [28-30].

Despite being a particle-based method, SPH has hitherto primarily been used to model continuous matter. For discontinuous matter, such as granular materials, the discrete element method (DEM) is instead commonly used. The DEM was proposed by Cundall and Strack more than 40 years ago [9] and is, like SPH, a Lagrangian particle-based method. However, contrary to SPH, the interparticle forces and torques are generally determined from the (normal and tangential) overlaps between particles, which represent local particle deformations. Hence, the deformation resulting from a certain contact is considered to be local in the sense that it does not affect the overall shape of the particle or the stiffness of any other contact(s) on the same particle. However, more general non-local contact models are emerging $[6,19,23,27]$.

It is interesting to note that the traditional SPH and the DEM thus are complementary in the sense that SPH considers only nonlocal forces and the DEM only local forces. These methods are also strongly related to peridynamics, developed by Stewart Silling at Sandia National Laboratories, which comes in two flavours: bond based [48] and state based [50]. In bond-based peridynamics, particles interact via forces directed along bonds between particles, i.e. via central forces. However, the assumption of central forces leads to severe restrictions of the class of materials that can be modelled using bond-based peridynamics. In particular, the Poisson ratio is always $1 / 4$ for isotropic materials [51]. For this reason, peridynamic states were introduced, which in effect result in a nonlocal coupling between bonds [50]. This means that arbitrary material models can be adapted for use within state-based peridynamics, as for SPH. In fact, it has been pointed out [17] that the commonly used numerical discretisation of state-based peridynamics [50] is equivalent to a total Lagrangian corrected SPH [5].

Acknowledging the complementary nature of SPH and the DEM, the purpose of this work is to take the first steps towards a macroscopically consistent discrete method for granular materials. Specifically, a particle-based method for continua is developed whose SPH-like component is based on the most widely accepted strain definition for granular media, a Delaunay-based strain developed by Bagi [1] and elaborated upon by others $[11,12,26]$. This strain definition is based on a decomposition of space into simplices and thus has much in common with the Delaunay Density Estimator Method proposed by Schaap and Weygaert [45], suggested for use in SPH by Pelupessy [41]. The DEM-like component is based on pairwise interactions between particles and produces a generalisation of the Ganzenmuller [16,18] hourglass stabilisation procedure to non-central forces. Aiming primarily for a description of solid materials, the energy corresponding to rotation of individual particles is disregarded, as is common practice in SPH. Although the starting points are distinct, it will be seen in the following that the resulting method has strong similarities with the one obtained by nodally integrated finite elements, in particular nodally averaged tetrahedral elements [10,42].

\section{Kinematics}

\subsection{Basic definitions}

We consider a system of discrete particles occupying a connected domain $\mathcal{D}$ and let lowercase letters in the beginning of the alphabet (e.g. $a, b$ and $c$ ) refer to particles. The reference placement of particle $a$ is $\boldsymbol{X}_{a}$ and its current position is $\boldsymbol{x}_{a}$. We assume the existence of particles located on the boundaries (boundary particles), such that the domain $\mathcal{D}$ can be decomposed into simplices via a triangulation (in 2D) or a tetrahedralisation (in 3D) in which the particles constitute the vertices; in effect, we thus assume the existence of an underlying triangular or tetrahedral mesh. We will adapt our language to the three-dimensional case but will, for simplicity, provide schematic illustrations in two dimensions, as in Fig. 1. The particles are assumed to interact via nearestneighbour interactions only, with bonds corresponding to edges of the underlying mesh. The vectors $\boldsymbol{X}_{a b}=\boldsymbol{X}_{b}-\boldsymbol{X}_{a}$ and $\boldsymbol{x}_{a b}=\boldsymbol{x}_{b}-\boldsymbol{x}_{a}$ point from the centre of particle $a$ to the centre of a neighbouring particle $b$ in the reference and current configurations, respectively. These are often referred to as branch vectors in the granular mechanics literature $[1,12]$. 


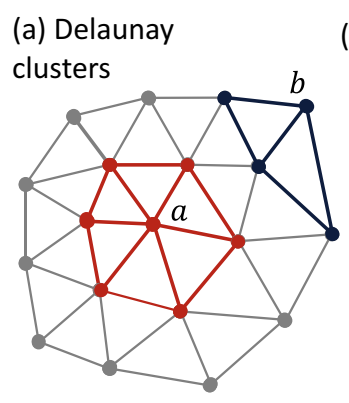

(b) Cluster volume $V_{a}^{(\mathrm{c})}$ and particle volume $V_{a}$

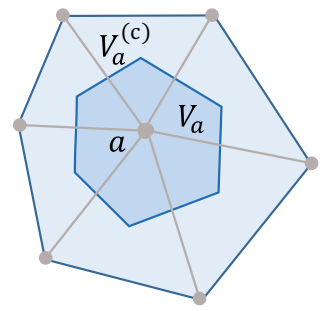

Fig. 1 a Definition of Delaunay clusters based on an underlying tesselation into simplices (triangles in 2D and tetrahedra in 3D). A Delaunay cluster for an internal particle labelled $a$ is shown in red and a cluster for a boundary particle labelled $b$ in blue. b Illustration of the Delaunay cluster volume $V_{a}^{(\mathrm{c})}$ and the particle volume $V_{a}$ (color online)

Since distinct simplices are disjoint, the total volume of the domain $\mathcal{D}$, denoted $V_{\text {tot }}$, can be obtained as

$V_{\text {tot }}=\operatorname{Vol} \mathcal{D}=\sum_{\alpha} \operatorname{Vol} \mathcal{S}_{\alpha}$

where the sum extends over all simplices $\mathcal{S}_{\alpha}$ in the system. For each particle $a$, we introduce the Delaunay cluster $\mathcal{C}_{a}$ as the union of all simplices that contain particle $a$, i.e. [45]

$\mathcal{C}_{a}=\bigcup_{\alpha} \mathcal{S}_{\alpha}$

where the union thus extends over the set of simplices that have particle $a$ as one of its vertices (Fig. 1a). The Delaunay cluster has been referred to as the contiguous Voronoi cell [45] but this denomination is not used here, since the Delaunay cluster represents a set of adjoint Delaunay simplices rather than a Voronoi cell. Since distinct simplices are disjoint, the volume of the Delaunay cluster is obtained as

$\operatorname{Vol} \mathcal{C}_{a}=\sum_{\alpha} \operatorname{Vol} \mathcal{S}_{\alpha}$

where the sum has the same extent as the union preceding equation. Since each simplex has $n+1$ nodes (where $n$ is the number of spatial dimensions), each simplex contributes to $n+1$ Delaunay clusters, implying that

$\sum_{a} \operatorname{Vol} \mathcal{C}_{a}=(n+1) V_{\mathrm{tot}}$,

where the sum extends over all particles in the system. For each particle, we will therefore define two volumes: Firstly the volume $V_{a}^{(\mathrm{c})}=\operatorname{Vol} \mathcal{C}_{a}$ and secondly the volume $V_{a}=\operatorname{Vol} \mathcal{C}_{a} /(n+1)$. We will refer to $V_{a}^{(\mathrm{c})}$ as the Delaunay cluster volume and to $V_{a}$ as the particle volume. The difference between $V_{a}^{(\mathrm{c})}$ and $V_{a}$ is illustrated in Fig. $1 \mathrm{~b}$.

\subsection{Strain measures}

For each particle $a$, we define a discrete mean deformation gradient $\boldsymbol{F}_{a}$ as the average of the continuum deformation gradient,

$\boldsymbol{F}=\frac{\partial \varphi}{\partial \boldsymbol{X}}=\operatorname{Grad} \boldsymbol{\varphi}$

over a suitably selected volume containing particle $a$. Here $\boldsymbol{x}=\boldsymbol{\varphi}(X, t)$ is a motion, depending on the reference placement $X$ of a material particle and time $t$. As noted by Bagi [1], Schaap [45] and He [26], the Delaunay cluster represents a natural choice (at least as long as a total Lagrangian formulation is sought). The details of the derivation are deferred to Sect. 4.

Similarly, we introduce a mean deformation gradient for each particle pair $a b$ as

$\boldsymbol{F}_{a b}=\frac{1}{2}\left(\boldsymbol{F}_{a}+\boldsymbol{F}_{b}\right)$,

where an unweighed average is used for simplicity. The mean deformation gradient $\boldsymbol{F}_{a b}$ maps the referential branch vectors $\boldsymbol{X}_{a b}$ to $\boldsymbol{y}_{a b}=\boldsymbol{F}_{a b} \boldsymbol{X}_{a b}$. Note that $\boldsymbol{y}_{a b} \neq \boldsymbol{x}_{a b}$ in general, because $\boldsymbol{F}_{a b}$ only accounts for the affine particle deformation. As a measure of non-affine deformation of the bond between particles $a$ and $b$, we introduce a local displacement vector $\boldsymbol{u}_{a b}^{\text {loc }}$, defined as

$\boldsymbol{u}_{a b}^{\mathrm{loc}}=\boldsymbol{x}_{a b}-\boldsymbol{y}_{a b}=\boldsymbol{x}_{a b}-\boldsymbol{F}_{a b} \boldsymbol{X}_{a b}$.

A geometric interpretation of the local displacement vector is provided in Fig. 2. It can be compared to the corresponding total displacement vector

$\boldsymbol{u}_{a b}^{\mathrm{tot}}=\boldsymbol{x}_{a b}-\boldsymbol{X}_{a b}$.

It will also be convenient to introduce the unit normal vectors

$\hat{\boldsymbol{n}}_{a b}=\frac{\boldsymbol{x}_{a b}}{\left|\boldsymbol{x}_{a b}\right|}$

so that the (total or local) displacement $\boldsymbol{u}_{a b}$ can be decomposed into normal and tangential parts,

$\boldsymbol{u}_{a b}^{\mathrm{n}}=\left(\boldsymbol{u}_{a b} \cdot \hat{\boldsymbol{n}}_{a b}\right) \hat{\boldsymbol{n}}_{a b}=\frac{\left(\boldsymbol{u}_{a b} \cdot \boldsymbol{x}_{a b}\right)}{\left|\boldsymbol{x}_{a b}\right|^{2}} \boldsymbol{x}_{a b}$

and

$\boldsymbol{u}_{a b}^{\mathrm{t}}=\boldsymbol{u}_{a b}-\boldsymbol{u}_{a b}^{\mathrm{n}}$,

respectively. 
Fig. 2 a Definition of referential contact vectors $\boldsymbol{X}_{a b} / 2$ and

$\boldsymbol{X}_{b a} / 2$ from branch vectors

$\boldsymbol{X}_{a b}=-\boldsymbol{X}_{b a}=\boldsymbol{X}_{b}-\boldsymbol{X}_{a}$,

where $\boldsymbol{X}_{a}$ and $\boldsymbol{X}_{b}$ are the

referential placements of

particles $a$ and $b$. b Definition of

the local displacement vector

$\boldsymbol{u}_{a b}^{\mathrm{loc}}$ from the current particle

coordinates $\boldsymbol{x}_{a}$ and $\boldsymbol{x}_{b}$ and the

images $\boldsymbol{F}_{a} \boldsymbol{X}_{a b} / 2$ and $\boldsymbol{F}_{b} \boldsymbol{X}_{b a} / 2$

of the referential contact vectors under the affine transformations described by $\boldsymbol{F}_{a}$ and $\boldsymbol{F}_{b}$ (indicated by dashed lines)

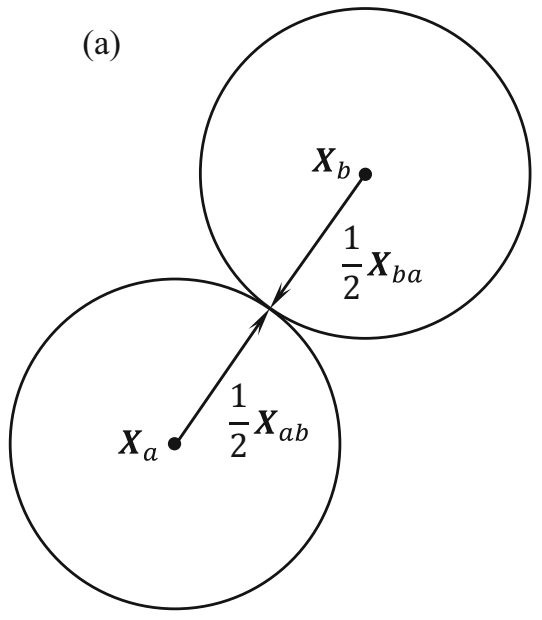

(b)

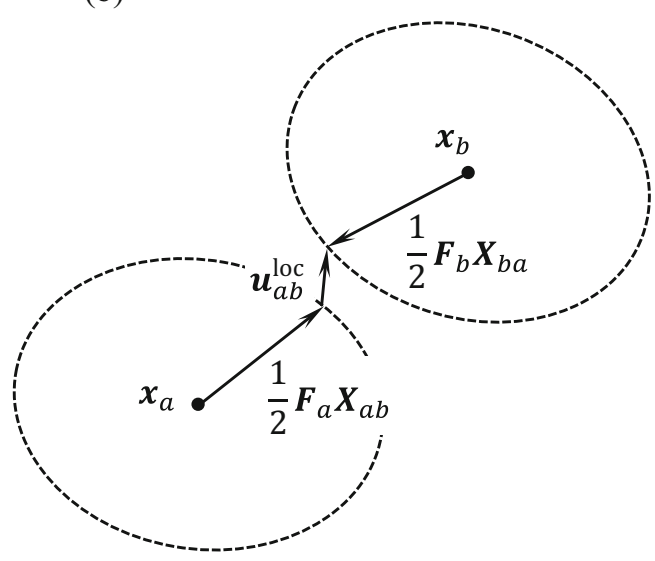

\section{Variational total Lagrangian formulation}

In order to derive the equations of motion, we here consider a conservative system, for which the discrete Lagrangian $\mathcal{L}$ takes the form (see, e.g. [22])

$\mathcal{L}=\mathcal{T}-\mathcal{V}$

The total kinetic energy $\mathcal{T}$ is expressed as

$\mathcal{T}=\frac{1}{2} \sum_{a} m_{a} v_{a}^{2}$

where $m_{a}$ and $v_{a}=\left|\boldsymbol{v}_{a}\right|$ are the mass and velocity of particle $a$, respectively. For simplicity, the energy corresponding to rotation of individual particles is thus disregarded, as is common practice in SPH. The potential energy $\mathcal{V}$ is considered to be the sum of three terms:

1. The internal (strain) energy $\mathcal{V}^{\text {int }}$ resulting from the global (affine) particle deformation, characterised by the mean deformation gradient $\boldsymbol{F}_{a}$. Hence,

$\mathcal{V}^{\text {int }}=\sum_{a} V_{a} U_{a}\left(\boldsymbol{F}_{a}\right)$

where $U_{a}$ is the internal energy per unit volume in the reference state and $V_{a}$ is the initial volume attributed to particle $a$. This term is commonly included in SPH (but not in the DEM).

2. The internal (strain) energy $\mathcal{V}^{\text {cnt }}$ resulting from local (non-affine) deformation at each contact. Noting that both the local displacement vector $\boldsymbol{u}_{a b}^{\text {loc }}$ and its normal and tangential components can be expressed in terms of $\boldsymbol{x}_{a b}$ and $\boldsymbol{y}_{a b}$ [compare Eqs. (7), (10) and (11)], we write

$\mathcal{V}^{\mathrm{cnt}}=\sum_{a<b} U_{a b}\left(\boldsymbol{x}_{a b}, \boldsymbol{y}_{a b}\right)$.
Here, $U_{a b}$ represents the pairwise interaction energy and the sum extends over all pairs of nearest neighbours $a$ and $b$. This term is typically not included in SPH, but the stabilisation of SPH proposed by Ganzenmuller [17] is of this form, albeit restricted to normal forces. It is also related to the stabilisation of state-based peridynamics models proposed by Littlewood [33] and Silling [49]. The DEM does, on the other hand, rely on pairwise contact forces, but inferred from the total rather than the local displacement [c.f. Eqs. (8) and (7)].

3. The external energy $\mathcal{V}^{\text {ext }}$, which for simplicity here is stated for the action of a constant gravitational force $g$,

$\mathcal{V}^{\mathrm{ext}}=-\sum_{a} m_{a} \boldsymbol{x}_{a} \cdot \boldsymbol{g}$.

The Euler-Lagrange equations (for particle $a$ ) take the form (see, e.g. [22])

$\frac{\mathrm{d}}{\mathrm{d} t}\left(\frac{\partial \mathcal{L}}{\partial \boldsymbol{v}_{a}}\right)-\frac{\partial \mathcal{L}}{\partial \boldsymbol{x}_{a}}=\mathbf{0}$.

Hence, introducing the canonical momentum

$\frac{\partial \mathcal{L}}{\partial \boldsymbol{v}_{a}}=\frac{\partial \mathcal{T}}{\partial \boldsymbol{v}_{a}}=m_{a} \boldsymbol{v}_{a}$,

together with the internal, contact and external forces,

$\boldsymbol{f}_{a}^{\mathrm{int}}=-\frac{\partial \mathcal{V}^{\text {int }}}{\partial \boldsymbol{x}_{a}}$,

$\boldsymbol{f}_{a}^{\mathrm{cnt}}=-\frac{\partial \mathcal{V}^{\mathrm{cnt}}}{\partial \boldsymbol{x}_{a}}$,

$\boldsymbol{f}_{a}^{\mathrm{ext}}=-\frac{\partial \mathcal{V}^{\mathrm{ext}}}{\partial \boldsymbol{x}_{a}}$, 
one obtains the equations of motion in the familiar form

$m_{a} \frac{\mathrm{d} \boldsymbol{v}_{a}}{\mathrm{~d} t}=\boldsymbol{f}_{a}^{\mathrm{int}}+\boldsymbol{f}_{a}^{\mathrm{cnt}}+\boldsymbol{f}_{a}^{\mathrm{ext}}$

The external force $f_{a}^{\text {ext }}$ is immediately obtained as

$\boldsymbol{f}_{a}^{\mathrm{ext}}=-\frac{\partial \mathcal{V}^{\mathrm{ext}}}{\partial \boldsymbol{x}_{a}}=m_{a} \boldsymbol{g}$.

The internal and contact forces ( $\boldsymbol{f}_{a}^{\mathrm{int}}$ and $\boldsymbol{f}_{a}^{\mathrm{cnt}}$, respectively) will be elaborated upon once the mean deformation gradient has been defined.

\section{The mean deformation gradient}

As already mentioned, we define the discrete mean deformation gradient $\boldsymbol{F}_{a}$ as the average of the continuum deformation gradient (5) over the corresponding Delaunay cluster. To simplify the ensuing developments, we introduce the following notation, illustrated in Fig. 3: First, for a given particle $a$, we let $\mathcal{P}_{a}$ denote the set of all particles that form the vertices of the associated Delaunay cluster $\mathcal{C}_{a}$. Hence, for $a$ not on the boundary, $\mathcal{P}_{a}$ corresponds to the set of nearest neighbours of $a$. For $a$ on the boundary, $\mathcal{P}_{a}$ in addition contains

(a) $\mathcal{P}_{a}$ and $\mathcal{P}_{b}$

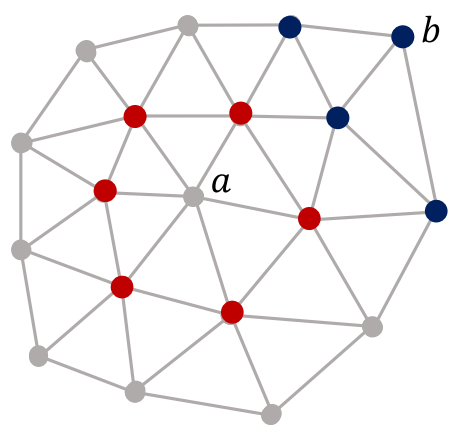

(c) $\mathcal{F}_{a}$ and $\mathcal{F}_{b}$

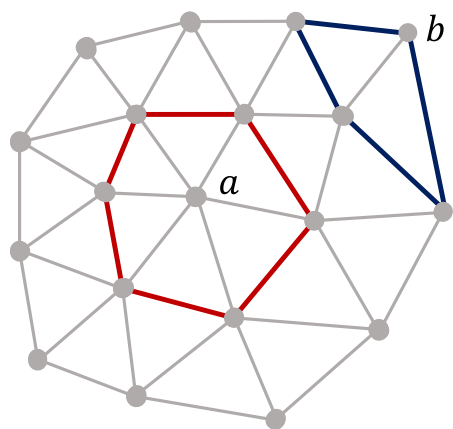

Fig. 3 Definition of the sets of particles $\mathbf{a} \mathcal{P}_{a}$ and $\mathbf{b} \mathcal{P}_{a \beta}$ and of faces $\mathbf{c}$ $\mathcal{F}_{a}$ and $\mathbf{d} \mathcal{F}_{a b}$ described in the text (color online) particle $a$ itself. Occasionally, it will be convenient to refer to the proper set of nearest neighbours of a certain particle $a$, which we denote as $\mathcal{P}_{a}^{\prime}$. For particle $a$ on the boundary, $\mathcal{P}_{a}^{\prime}$ thus contains all particles in $\mathcal{P}_{a}$ except particle $a$ itself. For particle $a$ not on the boundary, $\mathcal{P}_{a}^{\prime}$ and $\mathcal{P}_{a}$ coincide. Second, for a given face $\beta$ of a Delaunay cluster $\mathcal{C}_{a}$, we let $\mathcal{P}_{a \beta}$ denote the set of particles that are located at the vertices of the face. Third, for a given particle $a$, we let $\mathcal{F}_{a}$ denote the set of all faces of the associated Delaunay cluster $\mathcal{C}_{a}$. Fourth, for a given particle $b$ of a Delaunay cluster $\mathcal{C}_{a}$, we let $\mathcal{F}_{a b}$ denote the set of faces of $\mathcal{C}_{a}$ that have particle $b$ as one of its vertices. With this notation in hand, we proceed with the derivation of the mean deformation gradient.

Using Eq. (5) and the divergence theorem, we obtain

$$
\begin{aligned}
\boldsymbol{F}_{a} & =\frac{1}{V_{a}^{(\mathrm{c})}} \int_{\mathcal{C}_{a}} \boldsymbol{F} \mathrm{d} V=\frac{1}{V_{a}^{(\mathrm{c})}} \int_{\mathcal{C}_{a}} \operatorname{Grad} \boldsymbol{\varphi} \mathrm{d} V \\
& =\frac{1}{V_{a}^{(\mathrm{c})}} \int_{\partial \mathcal{C}_{a}} \boldsymbol{\varphi} \otimes \hat{\boldsymbol{N}} \mathrm{d} A,
\end{aligned}
$$

where $\hat{N}$ is the referential unit outward normal to the surface $\partial \mathcal{C}_{a}$ of $\mathcal{C}_{a}$. We let $\partial \mathcal{C}_{a \beta}$ denote the planar faces that between them form $\partial \mathcal{C}_{a}$, so that we can write

$\boldsymbol{F}_{a}=\frac{1}{V_{a}^{(\mathrm{c})}} \sum_{\beta \in \mathcal{F}_{a}} \int_{\partial \mathcal{C}_{a \beta}} \varphi \otimes \hat{N} \mathrm{~d} A$

Since $\hat{N}$ is constant in each of the integrals in Eq. (23), it suffices to integrate $\varphi$ over the faces $\partial \mathcal{C}_{a \beta}$. Assuming that $\varphi(X, t)$ varies linearly between the values $\varphi\left(X_{b}, t\right)$ at the vertices, we obtain

$\overline{\boldsymbol{x}}_{a \beta}=\frac{1}{A_{a \beta}} \int_{\partial \mathcal{C}_{a \beta}} \boldsymbol{\varphi d} A=\frac{1}{n} \sum_{b \in \mathcal{P}_{a \beta}} \varphi\left(X_{b}, t\right)=\frac{1}{n} \sum_{b \in \mathcal{P}_{a \beta}} \boldsymbol{x}_{b}$,

where $A_{a \beta}$ is the area of the face $\partial \mathcal{C}_{a \beta}$. Using Eq. (24) and introducing the referential area vector $\boldsymbol{A}_{a \beta}=A_{a \beta} \hat{\boldsymbol{N}}_{a \beta}$, Eq. (23) takes the form

$\boldsymbol{F}_{a}=\frac{1}{V_{a}^{(\mathrm{c})}} \sum_{\beta \in \mathcal{F}_{a}} \overline{\boldsymbol{x}}_{a \beta} \otimes \boldsymbol{A}_{a \beta}$

Substituting Eq. (24) in Eq. (25) and interchanging the order of summations, we obtain

$$
\begin{aligned}
\boldsymbol{F}_{a} & =\frac{1}{n V_{a}^{(\mathrm{c})}} \sum_{\beta \in \mathcal{F}_{a}} \sum_{b \in \mathcal{P}_{a \beta}} \boldsymbol{x}_{b} \otimes \boldsymbol{A}_{a \beta} \\
& =\frac{1}{n V_{a}^{(\mathrm{c})}} \sum_{b \in \mathcal{P}_{a}} \sum_{\beta \in \mathcal{F}_{a b}} \boldsymbol{x}_{b} \otimes \boldsymbol{A}_{a \beta} .
\end{aligned}
$$


Introduction of the referential vector

$\boldsymbol{B}_{a b}=\frac{1}{n(n+1)} \sum_{\beta \in \mathcal{F}_{a b}} \boldsymbol{A}_{a \beta}$

yields the final result

$\boldsymbol{F}_{a}=\frac{1}{V_{a}} \sum_{b \in \mathcal{P}_{a}} \boldsymbol{x}_{b} \otimes \boldsymbol{B}_{a b}$

where we have used the fact that $V_{a}=V_{a}^{(\mathrm{c})} /(n+1)$. The thus defined vector $\boldsymbol{B}_{a b}$ has been referred to as the complementary area vector by Bagi [1]. However, we will henceforth refer to $\boldsymbol{B}_{a b}$ as the gradient vector, because of its strong similarity to the gradient vectors commonly employed in SPH (see below).

The gradient vectors exhibit a number of useful properties, as we next demonstrate. First, summation over the second index yields

$$
\begin{aligned}
\sum_{b \in \mathcal{P}_{a}} \boldsymbol{B}_{a b} & =\frac{1}{n(n+1)} \sum_{b \in \mathcal{P}_{a}} \sum_{\beta \in \mathcal{F}_{a b}} \boldsymbol{A}_{a \beta} \\
& =\frac{1}{n+1} \sum_{\beta \in \mathcal{F}_{a}} \boldsymbol{A}_{a \beta}=\mathbf{0}
\end{aligned}
$$

since the sum of outwards directed area vectors over any closed surface vanishes according to the divergence theorem. Similarly, provided that not both particle $a$ and $b$ are on the boundary of the domain $\mathcal{D}$,

$\boldsymbol{B}_{a b}+\boldsymbol{B}_{b a}=\frac{1}{n(n+1)}\left(\sum_{\beta \in \mathcal{F}_{a b}} \boldsymbol{A}_{a \beta}+\sum_{\alpha \in \mathcal{F}_{b a}} \boldsymbol{A}_{b \alpha}\right)=\mathbf{0}$

because the parenthesis contains a sum of outwards directed area vectors over a closed surface, which vanishes. Provided that not both particle $a$ and $b$ are on the boundary of the domain $\mathcal{D}$, we thus from Eqs. (29) and (30) conclude that the gradient vectors are antisymmetric and that the sum over either index vanishes, i.e. that $\boldsymbol{B}_{b a}=-\boldsymbol{B}_{a b}$ and that

$\sum_{a \in \mathcal{P}_{b}} \boldsymbol{B}_{a b}=\sum_{b \in \mathcal{P}_{a}} \boldsymbol{B}_{a b}=\mathbf{0}$.

Equation (29) enables us to write Eq. (28) in the more symmetric form

$\boldsymbol{F}_{a}=\frac{1}{V_{a}} \sum_{b \in \mathcal{P}_{a}} \boldsymbol{x}_{a b} \otimes \boldsymbol{B}_{a b}$ where $\boldsymbol{x}_{a b}=\boldsymbol{x}_{b}-\boldsymbol{x}_{a}$ is the current branch vector for particles $a$ and $b$. Since the deformation gradient reduces to the identity tensor $\boldsymbol{I}$ when the reference coordinates are substituted for the current coordinates in either of Eqs. (28) or (32), we immediately obtain the following duality relation

$$
\frac{1}{V_{a}} \sum_{b \in \mathcal{P}_{a}} \boldsymbol{X}_{b} \otimes \boldsymbol{B}_{a b}=\frac{1}{V_{a}} \sum_{b \in \mathcal{P}_{a}} \boldsymbol{X}_{a b} \otimes \boldsymbol{B}_{a b}=\boldsymbol{I}
$$

where $\boldsymbol{X}_{a b}=\boldsymbol{X}_{b}-\boldsymbol{X}_{a}$ is the referential branch vector.

Noting that the gradient vectors are referential and hence time independent, one immediately obtains

$\dot{\boldsymbol{F}}_{a}=\frac{1}{V_{a}} \sum_{b \in \mathcal{P}_{a}} \dot{\boldsymbol{x}}_{b} \otimes \boldsymbol{B}_{a b}=\frac{1}{V_{a}} \sum_{b \in \mathcal{P}_{a}} \boldsymbol{v}_{b} \otimes \boldsymbol{B}_{a b}$

where the superposed dot denotes the material time derivative and where $\boldsymbol{v}_{b}$ is the velocity of particle $b$. Hence, the velocity gradient $\boldsymbol{l}_{a}$ and the rate of deformation tensor $\boldsymbol{d}_{a}$ (see, e.g. [25]) can be determined as

$\boldsymbol{l}_{a}=\dot{\boldsymbol{F}}_{a} \boldsymbol{F}_{a}^{-1}$

and

$\boldsymbol{d}_{a}=\frac{1}{2}\left(\boldsymbol{l}_{a}+\boldsymbol{l}_{a}^{\mathrm{T}}\right)$,

respectively. The rate of deformation tensor can be used, e.g. to define viscous stresses.

\section{Force evaluation}

\subsection{Preliminaries}

For future reference, we notice that

$\frac{\partial \boldsymbol{x}_{b c}}{\partial \boldsymbol{x}_{a}}=\left(\delta_{c a}-\delta_{b a}\right) \boldsymbol{I}$,

where $\delta_{c a}$ is the Kronecker delta. Using Eqs. (6) and (28) and the antisymmetry of $\boldsymbol{X}_{b c}$ (i.e. that $\boldsymbol{X}_{b c}=-\boldsymbol{X}_{c b}$ ), we may expand $\boldsymbol{y}_{b c}=\boldsymbol{F}_{b c} \boldsymbol{X}_{b c}$ as

$$
\begin{aligned}
\boldsymbol{y}_{b c} & =\frac{1}{2}\left(\boldsymbol{F}_{b} \boldsymbol{X}_{b c}-\boldsymbol{F}_{c} \boldsymbol{X}_{c b}\right) \\
& =\frac{1}{2}\left(\sum_{d \in \mathcal{P}_{b}} H_{b c d} \boldsymbol{x}_{d}-\sum_{d \in \mathcal{P}_{c}} H_{c b d} \boldsymbol{x}_{d}\right)
\end{aligned}
$$

where

$H_{b c d}=\frac{\boldsymbol{X}_{b c} \cdot \boldsymbol{B}_{b d}}{V_{b}}$. 
Since $H_{a b c}$ is referential and thus independent of $\boldsymbol{x}_{a}$, this yields

$\frac{\partial \boldsymbol{y}_{b c}}{\partial \boldsymbol{x}_{a}}=\frac{1}{2}\left(H_{b c a}-H_{c b a}\right) \boldsymbol{I}$.

In order to evaluate the derivative of the mean deformation gradient, we resort to an orthonormal Cartesian coordinate system, with components labelled by $i, j$ and $k$. Since $\boldsymbol{B}_{b c}$ is referential, we find using Eqs. (28) that

$\frac{\partial F_{j k}^{b}}{\partial x_{i}^{a}}=\frac{1}{V_{b}} \sum_{c} \frac{\partial x_{j}^{c}}{\partial x_{i}^{a}} B_{k}^{b c}=\frac{1}{V_{b}} \sum_{c} \delta_{j i} \delta_{c a} B_{k}^{b c}=\frac{1}{V_{b}} \delta_{i j} B_{k}^{b a}$

where the properties of the Kronecker delta have been used.

\subsection{Internal force}

From Eq. (19) and the chain rule, the internal force is obtained as

$$
\begin{aligned}
\boldsymbol{f}_{a}^{\text {int }} & =-\frac{\partial \mathcal{V}^{\text {int }}}{\partial \boldsymbol{x}_{a}}=-\sum_{b} V_{b} \frac{\partial U_{b}}{\partial \boldsymbol{F}_{b}}: \frac{\partial \boldsymbol{F}_{b}}{\partial \boldsymbol{x}_{a}} \\
& =-\sum_{b} V_{b} \boldsymbol{P}_{b}: \frac{\partial \boldsymbol{F}_{b}}{\partial \boldsymbol{x}_{a}}
\end{aligned}
$$

where the colon indicates double contraction and where

$\boldsymbol{P}_{b}=\frac{\partial U_{b}}{\partial \boldsymbol{F}_{b}}$

is the first Piola-Kirchhoff stress tensor for particle $b$. Using Eq. (41) in Eq. (42), expressed in component form, we obtain

$\left(f^{\text {int }}\right)_{i}^{a}=-\sum_{b} V_{b} P_{j k}^{b} \frac{\partial F_{j k}^{b}}{\partial x_{i}^{a}}=-\sum_{b} P_{i k}^{b} B_{k}^{b a}$.

Hence, in direct notation,

$\boldsymbol{f}_{a}^{\text {int }}=-\sum_{b \in \mathcal{P}_{a}} \boldsymbol{P}_{b} \boldsymbol{B}_{b a}$,

where we have explicitly indicated that the sum is to be taken over the set of nearest neighbours to particle $a$ (including particle $a$ itself if the particle is located on the boundary).

One can note that the internal forces vanish for a constant stress field provided that the sum of the gradient vectors over the first index vanishes. Hence, fulfilment of Eq. (31) for particles not on the boundary is required for satisfaction of the patch test. This is nothing else than the integration constraint derived by Chen et al. [7] (see also [42]).
Each term can be made antisymmetric as follows:

$\boldsymbol{f}_{a}^{\mathrm{int}}=\sum_{b \in \mathcal{P}_{a}}\left(\boldsymbol{P}_{a} \boldsymbol{B}_{a b}-\boldsymbol{P}_{b} \boldsymbol{B}_{b a}\right)$

since the sum of the added terms vanishes as a result of Eq. (29). Hence, we may interpret

$\boldsymbol{f}_{a b}^{\mathrm{int}}=\boldsymbol{P}_{a} \boldsymbol{B}_{a b}-\boldsymbol{P}_{b} \boldsymbol{B}_{b a}$.

as the contribution to the internal force on particle $a$ due to contact with particle $b$.

\subsection{Contact force}

From Eq. (19) and the chain rule, the contact force is obtained as

$$
\begin{aligned}
\boldsymbol{f}_{a}^{\mathrm{cnt}} & =-\frac{\partial \mathcal{V}^{\mathrm{cnt}}}{\partial \boldsymbol{x}_{a}}=-\sum_{b<c} \frac{\partial U_{b c}}{\partial \boldsymbol{x}_{a}} \\
& =-\sum_{b<c}\left(\frac{\partial U_{b c}}{\partial \boldsymbol{x}_{b c}} \cdot \frac{\partial \boldsymbol{x}_{b c}}{\partial \boldsymbol{x}_{a}}+\frac{\partial U_{b c}}{\partial \boldsymbol{y}_{b c}} \cdot \frac{\partial \boldsymbol{y}_{b c}}{\partial \boldsymbol{x}_{a}}\right)
\end{aligned}
$$

Inserting the expressions for $\partial \boldsymbol{x}_{b c} / \partial \boldsymbol{x}_{a}$ and $\partial \boldsymbol{y}_{b c} / \partial \boldsymbol{x}_{a}$ provided by Eqs. (37) and (40), respectively, one obtains

$\boldsymbol{f}_{a}^{\mathrm{cnt}}=-\sum_{b<c}\left[\left(\delta_{c a}-\delta_{b a}\right) \frac{\partial U_{b c}}{\partial \boldsymbol{x}_{b c}}+\frac{1}{2}\left(H_{b c a}-H_{c b a}\right) \frac{\partial U_{b c}}{\partial \boldsymbol{y}_{b c}}\right]$

Noting that the derivative $\partial U_{b c} / \partial \boldsymbol{x}_{b c}$ and $\partial U_{b c} / \partial \boldsymbol{y}_{b c}$ and the pre-factors $\delta_{c a}-\delta_{b a}$ and $H_{b c a}-H_{c b a}$ all change sign when $b$ and $c$ are interchanged, the sum over all particle pairs $b<c$ is converted to a double sum over nearest neighbours $b$ and $c$. Moreover, exploiting the properties of the Kronecker delta, the final result is obtained as

$\boldsymbol{f}_{a}^{\mathrm{cnt}}=\sum_{b \in \mathcal{P}_{a}^{\prime}} \frac{\partial U_{a b}}{\partial \boldsymbol{x}_{a b}}-\sum_{b \in \mathcal{P}_{a}} \sum_{c \in \mathcal{P}_{b}^{\prime}} \tilde{H}_{b c a} \frac{\partial U_{b c}}{\partial \boldsymbol{y}_{b c}}$,

where $\tilde{H}_{b c a}=H_{b c a} / 2$ has been introduced for convenience. Here we have written $\mathcal{P}_{a}^{\prime}$ and $\mathcal{P}_{b}^{\prime}$ to indicate the proper set of nearest neighbours of $a$ and $b$, respectively.

\section{Constitutive equations}

\subsection{Material model}

We assume a neo-Hookean material model with strain energy

$U_{b}^{\text {int }}=\frac{\lambda}{2}\left(\ln J_{b}\right)^{2}-\mu \ln J_{b}+\frac{\mu}{2}\left(\operatorname{tr} C_{b}-3\right)$ 
where $\boldsymbol{C}_{b}=\boldsymbol{F}_{b}^{\mathrm{T}} \boldsymbol{F}_{b}$ is the mean right Cauchy-Green deformation tensor for particle $b$ and $\boldsymbol{J}_{b}=\operatorname{det} \boldsymbol{F}_{\boldsymbol{b}}=\left(\operatorname{det} \boldsymbol{C}_{b}\right)^{1 / 2}$. Using standard solid mechanics results (see, e.g. [25]) one obtains the second Piola-Kirchhoff stress tensor $\boldsymbol{S}_{b}$ as

$\boldsymbol{S}_{b}=2 \frac{\partial U_{b}^{\text {int }}}{\partial \boldsymbol{C}_{b}}=\left(\lambda \ln J_{b}-\mu\right) \boldsymbol{C}_{b}^{-\mathrm{T}}+\mu \boldsymbol{I}$

where $\boldsymbol{C}_{b}^{-\mathrm{T}}$ is the transpose of the inverse right CauchyGreen deformation tensor and $\boldsymbol{I}$ is the second-order identity tensor. Moreover, the first Piola-Kirchhoff stress tensor $\boldsymbol{P}_{b}$ is obtained as

$\boldsymbol{P}_{b}=\boldsymbol{F}_{b} \boldsymbol{S}_{b}=\left(\lambda \ln J_{b}-\mu\right) \boldsymbol{F}_{b}^{-\mathrm{T}}+\mu \boldsymbol{F}_{b}$.

The first Piola-Kirchhoff stress is used to calculate the internal force via Eq. (45). The Lamé parameters may be expressed as

$\lambda=\frac{v E}{(1+v)(1-2 v)}$ and $\mu=\frac{E}{2(1+v)}$

where $E$ is Young's modulus and $v$ Poisson's ratio. We also note that the longitudinal (primary) and the transverse (secondary) wave speeds are obtained as

$c_{\mathrm{p}}=\sqrt{\frac{\lambda+2 \mu}{\rho}}$ and $c_{\mathrm{s}}=\sqrt{\frac{\mu}{\rho}}$,

respectively.

\subsection{Contact model}

We will here assume a linearly elastic contact model with normal and tangential stiffness $K_{a b}^{\mathrm{n}}=K_{b a}^{\mathrm{n}}$ and $K_{a b}^{\mathrm{t}}=K_{b a}^{\mathrm{t}}$ for contact between the nearest neighbours $a$ and $b$. Hence the contact energy is written

$U_{a b}=\frac{1}{2} K_{a b}^{\mathrm{n}}\left|\boldsymbol{u}_{a b}^{\mathrm{loc}, \mathrm{n}}\right|^{2}+\frac{1}{2} K_{a b}^{\mathrm{t}}\left|\boldsymbol{u}_{a b}^{\mathrm{loc}, \mathrm{t}}\right|^{2}$

Using Eqs. (10) and (11), we thus obtain

$$
\begin{aligned}
\frac{\partial U_{b c}}{\partial \boldsymbol{x}_{b c}}= & K_{b c}^{\mathrm{n}} \boldsymbol{u}_{b c}^{\mathrm{loc}, \mathrm{n}}+K_{b c}^{\mathrm{t}} \boldsymbol{u}_{b c}^{\mathrm{loc}, \mathrm{t}} \\
& +\left(K_{b c}^{\mathrm{n}}-K_{b c}^{\mathrm{t}}\right) \frac{\left(\boldsymbol{u}_{b c}^{\mathrm{loc}} \cdot \boldsymbol{x}_{b c}\right)}{\left|\boldsymbol{x}_{b c}\right|^{2}} \boldsymbol{u}_{b c}^{\mathrm{loc}, \mathrm{t}}
\end{aligned}
$$

and

$\frac{\partial U_{b c}}{\partial \boldsymbol{y}_{b c}}=-\left(K_{b c}^{\mathrm{n}} \boldsymbol{u}_{b c}^{\mathrm{loc}, \mathrm{n}}+K_{b c}^{\mathrm{t}} \boldsymbol{u}_{b c}^{\mathrm{loc}, \mathrm{t}}\right)$.
In the special case that the normal and tangential contact stiffness coincide, i.e. when $K_{a b}^{\mathrm{n}}=K_{a b}^{\mathrm{t}}=K_{a b}$, the above equations reduce to

$$
\begin{gathered}
U_{a b}=\frac{1}{2} K_{a b}\left|\boldsymbol{u}_{a b}^{\mathrm{loc}}\right|^{2}, \frac{\partial U_{b c}}{\partial \boldsymbol{x}_{b c}}=K_{b c} \boldsymbol{u}_{b c}^{\mathrm{loc}} \\
\text { and } \frac{\partial U_{b c}}{\partial \boldsymbol{y}_{b c}}=-K_{b c} \boldsymbol{u}_{b c}^{\mathrm{loc}} .
\end{gathered}
$$

In this work, the normal and tangential contact stiffness are expressed as

$K_{b c}^{\mathrm{n}}=\xi_{\mathrm{n}} \frac{E A_{b c}}{L_{b c}}$ and $K_{b c}^{\mathrm{t}}=\xi_{\mathrm{t}} \frac{G A_{b c}}{L_{b c}}$

where $E$ is Young's modulus and $G=\mu$ is the shear modulus. Two geometric quantities are included, namely $A_{b c}=4 \times\left(\left|\boldsymbol{B}_{b c}\right|+\left|\boldsymbol{B}_{c b}\right|\right) / 2$, which is an estimate of the area of the interface between particle $b$ and $c$ [cf. Eq. (27)] and $L_{b c}=\left|\boldsymbol{X}_{b c}\right|$, which is the initial distance between particle $b$ and $c$. Finally, $\xi_{\mathrm{n}}$ and $\xi_{\mathrm{t}}$ are non-dimensional parameters.

\subsection{Artificial viscosity}

An artificial viscosity can be used to reduce oscillations in shock-dominated problems $[39,40]$. We here use a viscous (Cauchy) stress of the form [37]

$\boldsymbol{\sigma}^{\mathrm{visc}}=\rho\left(c_{1} c_{\mathrm{p}} h+c_{2} h^{2}|\operatorname{tr} \boldsymbol{d}|\right) \boldsymbol{d}$

where $c_{1}$ and $c_{2}$ are two non-dimensional constants, $c_{\mathrm{p}}$ the longitudinal wave speed and $h$ is a characteristic size, here taken as the minimum length of the referential branch vectors. The viscous stress is incorporated as an addition to the elastic first Piola-Kirchhoff stress, obtained as (see, e.g. [25])

$\boldsymbol{P}^{\mathrm{visc}}=J \boldsymbol{\sigma}^{\mathrm{visc}} \boldsymbol{F}^{-\mathrm{T}}=\rho \boldsymbol{J}\left(c_{1} c_{\mathrm{p}} h+c_{2} h^{2}|\operatorname{tr} \boldsymbol{d}|\right) \boldsymbol{d} \boldsymbol{F}^{-\mathrm{T}}$.

This type of dissipation can also be added constitutively, i.e. be considered to be part of the material rather than algorithmic behaviour [44]. When artificial viscosity is included, the sum of the elastic and viscous first Piola-Kirchhoff stress, Eqs. (53) and (62), is used to calculate the internal force via Eq. (45).

\section{Implementation details}

Based on the initial particle arrangement, a Delaunay tetrahedralisation was obtained using Tetgen, developed and maintained by $\mathrm{Si}$ [47]. As is commonly done in SPH [38], a 
standard leapfrog scheme was used to integrate the equations of motion in time. When damping was applied, the velocity was calculated as described in [15].

\section{Numerical tests and examples}

As described in Sect. 2.1, it is assumed that the computational domain can be decomposed into simplices with particles as vertices but the particle placement is otherwise arbitrary. In practice, however, it is preferable to avoid very short inter-particle distances, since the stable time step is proportional to the minimum particle separation when a Courant-Friedrichs-Lewy condition is used [8]. In all numerical tests and examples, the initial particle positions were based on a regular cubic lattice. In most of the examples, randomness was introduced by perturbing the particles in all directions, while respecting the location of faces and edges, by an amount not exceeding $25 \%$ of the regular particle spacing in each direction. The constitutive equations summarised in Sect. 6 were used throughout.

\subsection{Patch test}

The patch test in the forms described by Taylor et al. [53] was utilised to assess the basic characteristics of the proposed method. As illustrated in Fig. 4, an irregular arrangement of $4 \times 4 \times 4$ particles was used to represent a cubic specimen with a $10 \mathrm{~mm}$ side. For clarity, the particles are in this example displayed as red spheres and the bonds or contacts between

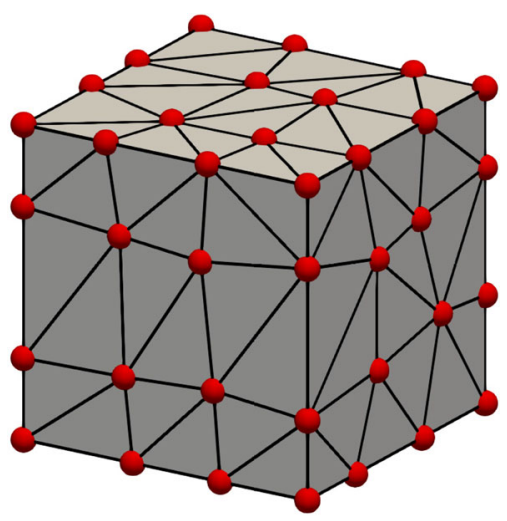

Fig. 4 Setup for the patch test. Particles are displayed as red spheres and bonds as black lines (color online)

Table 1 Results obtained from the patch test

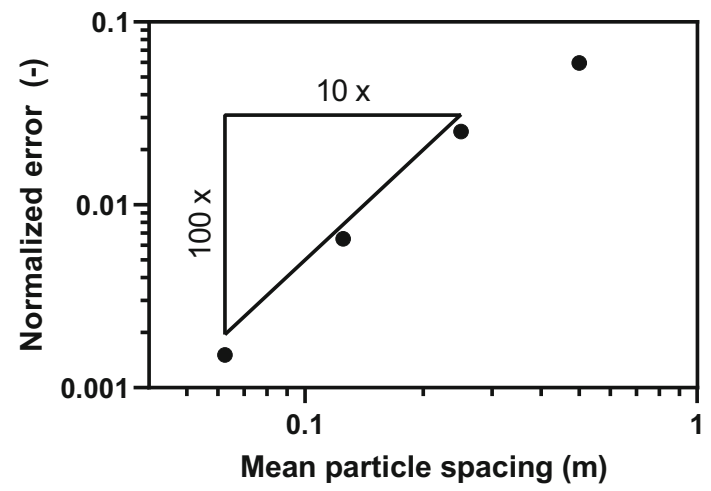

Fig. 5 Normalised displacement error (in the $L_{2}$ norm) versus mean particle spacing for the swinging-cube test

them as black solid lines. The material parameters are $E=$ $7.0 \mathrm{GPa}, v=0.25$ and $\rho=1.0 \times 10^{3} \mathrm{~kg} / \mathrm{m}^{3}$ and the contact parameters as set to unity (i.e. $\xi_{\mathrm{n}}=\xi_{\mathrm{t}}=1$ ). Following [55], the displacement field is postulated as

$\boldsymbol{u}=u_{0}\left(\begin{array}{l}2 X_{1}+X_{2}+X_{3} \\ X_{1}+2 X_{2}+X_{3} \\ X_{1}+X_{2}+2 X_{3}\end{array}\right)$

where $u_{0}=5 \times 10^{-4}$. In test $\mathrm{A}$, the displacement is prescribed for all particles and force equilibrium is tested for internal particles. In test B, the displacement is prescribed for all boundary particles, and the displacement error is determined for internal particles. In test $\mathrm{C}$, a minimal number of essential boundary conditions needed to avoid rigid body translation or rotation are enforced and the traction corresponding to the displacement field (63) is prescribed on all boundaries. Specifically, all displacement components were prescribed on one corner particle and the vertical displacement was prescribed on two additional corner particles. Since an explicit solver was used, the displacements and/or loads were applied gradually during $5 \mathrm{~ms}$ for tests $\mathrm{B}$ and $\mathrm{C}$ and damping was used to obtain a static solution. The results obtained are summarised in Table 1. The magnitude of the error is somewhat larger in test A than in tests B and C, because a minute error in displacement is translated into a significantly larger error in force as a result of the relatively high Young's modulus. Nevertheless, all three forms of the patch test are passed. This result is anticipated, since the gradient vectors fulfill the integration constraint $[7,42]$ expressed by Eq. (31).

\subsection{Swinging cube}

The purpose of this test is to assess the convergence of the proposed method in a dynamic three-dimensional setting. A cube with side-length $L=1 \mathrm{~m}$ is considered, occupying the referential domain $0 \leq X_{1}, X_{2}, X_{3} \leq 1 \mathrm{~m}$. Zero normal dis- 


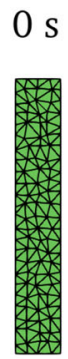

$0.5 \mathrm{~s}$
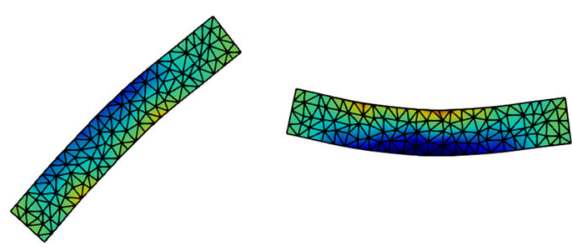

$1.5 \mathrm{~s}$

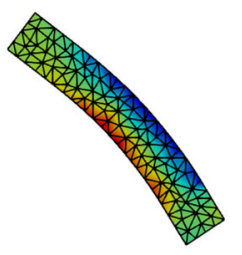

$2.0 \mathrm{~s}$

$2.5 \mathrm{~s}$

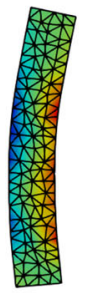

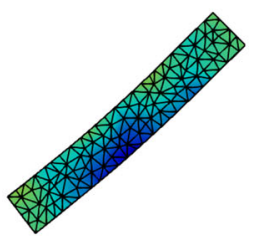

Pressure $(\mathrm{Pa})$

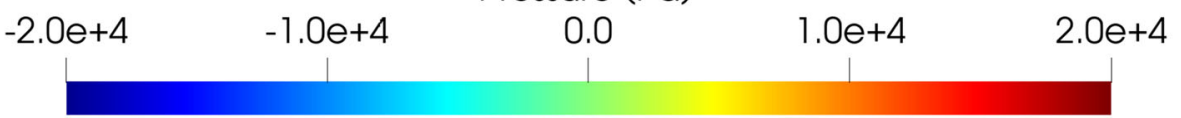

Fig. 6 Location and shape of free bending and rotating beam at the indicated times (color online)

placement is enforced for $X_{1}=X_{2}=X_{3}=0$ (symmetric boundary conditions) whereas zero tangential displacement is enforced for $X_{1}=X_{2}=X_{3}=1$ (anti-symmetric boundary conditions). The following analytic solution for the displacement field $\boldsymbol{u}$ is valid $[3,46]$

$$
\boldsymbol{u}=\cos (\omega t)\left[\begin{array}{l}
U_{1} \sin \left(k X_{1}\right) \cos \left(k X_{2}\right) \cos \left(k X_{3}\right) \\
U_{2} \cos \left(k X_{1}\right) \sin \left(k X_{2}\right) \cos \left(k X_{3}\right) \\
U_{3} \cos \left(k X_{1}\right) \cos \left(k X_{2}\right) \sin \left(k X_{3}\right)
\end{array}\right]
$$

provided that the magnitude of the oscillation is sufficiently small and that the amplitude coefficients fulfill the auxiliary condition $U_{1}+U_{2}+U_{3}=0$. Here, $\omega=\sqrt{3} \pi c_{\mathrm{s}} /(2 L)$ and $k=\pi /(2 L)$. Specifically, we let $U_{1}=U_{2}=5.0 \times 10^{-4} \mathrm{~m}$ and $U_{3}=-1.0 \times 10^{-3} \mathrm{~m}$ and prescribe the initial displacement (and velocity) according to Eq. (64). As previously done [3], the error of the numerical solution after $2 \times 10^{-3} \mathrm{~s}$ was benchmarked against the analytical result. The material parameters are $E=17.0 \mathrm{MPa}, v=0.3$ and $\rho=$ $1.1 \times 10^{3} \mathrm{~kg} / \mathrm{m}^{3}$ and the contact parameters are set to unity $\left(\xi_{\mathrm{n}}=\xi_{\mathrm{t}}=1\right)$. Figure 5 displays the normalised displacement error, measured in the $L_{2}$ norm, as a function of the mean particle spacing. The displacement is seen to exhibit a quadratic convergence towards the correct solution. This result is anticipated for a linearly consistent method, since the truncation error then will be of second order.

\subsection{Bending and rotation of free beam}

The purpose of this test is to assess the momentumpreservation properties of the proposed method. A beam of length $6 \mathrm{~m}$, with a square cross section (side length $1 \mathrm{~m}$ ) is considered, occupying the referential domain $-0.5 \mathrm{~m} \leq$ $X_{1}, X_{2} \leq 0.5 \mathrm{~m}$ and $0 \leq X_{3} \leq 6 \mathrm{~m}$. The beam is free, i.e. no tractions or displacements are prescribed. The initial velocity

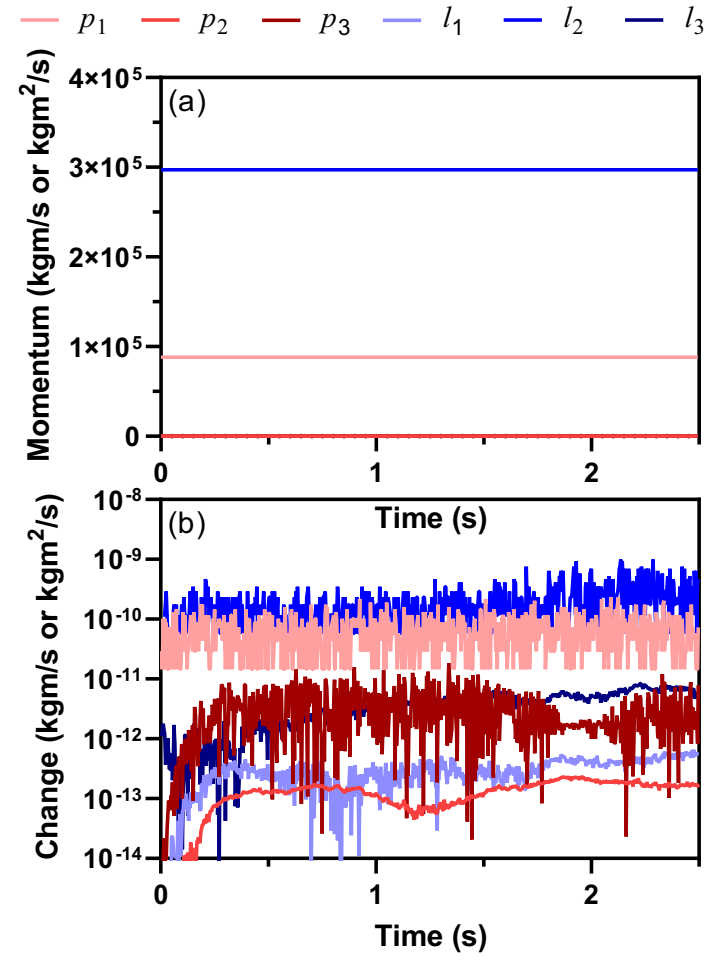

Fig. 7 a Components of linear and angular momentum (denoted by $p_{1}$, $p_{2}, p_{3}$ and $\ell_{1}, \ell_{2}, \ell_{3}$, respectively) and $\mathbf{b}$ magnitude of change in each momentum component from its initial value (color online)

is

$\boldsymbol{v}=v_{0}\left(\begin{array}{c}1+\left(\frac{X_{3}}{L}\right)^{2} \\ 0 \\ 0\end{array}\right)$,

where $v_{0}=10 \mathrm{~m} / \mathrm{s}$ (the superposed constant velocity is used to more clearly display the location of the beam at various times). The material parameters are $E=1.0 \mathrm{MPa}, v=0.3$ and $\rho=1.1 \times 10^{3} \mathrm{~kg} / \mathrm{m}^{3}$ and the contact parameters as 


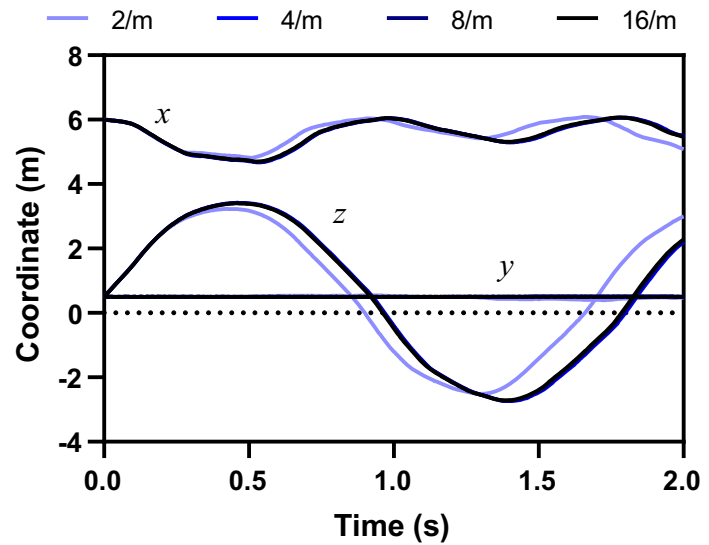

Fig. 8 Coordinates of the top right free corner of an oscillating cantilever beam as a function of time for four different mean particle densities (color online)

set to unity $\left(\xi_{\mathrm{n}}=\xi_{\mathrm{t}}=1\right)$. The nonzero linear and angular momentum components (with respect to the origin) can readily be calculated analytically as $p_{1}=8.8 \times 10^{4} \mathrm{~kg} \mathrm{~m} / \mathrm{s}$ and $\ell_{2}=2.97 \times 10^{5} \mathrm{~kg} \mathrm{~m}^{2} / \mathrm{s}$. The location and shape of the beam at certain instants of time are displayed in Fig. 6 . Since the Young's modulus is relatively small, the beam is seen to undergo a pronounced bending in addition to translational and rotational motion. In Fig. 7a, the linear and angular momentum components are shown as a function of time. As a result of collocation of mass, the numerically computed momentum components differed slightly from their analytical counterparts (e.g. $p_{1} \approx 8.802 \times 10^{3} \mathrm{~kg} \mathrm{~m} / \mathrm{s}$ and $\ell_{2} \approx 2.972 \times 10^{5} \mathrm{~kg} \mathrm{~m}^{2} / \mathrm{s}$ initially), but this difference is too small to be noticeable in the figure. In Fig. 7b, the magnitude of the change in each momentum component from its initial value is displayed on a logarithmic scale. The magnitude of the change is $\lesssim 1 \times 10^{-9} \mathrm{~kg} \mathrm{~m}^{2} / \mathrm{s}$ for $\ell_{2}$, $\lesssim 1 \times 10^{-10} \mathrm{~kg} \mathrm{~m} / \mathrm{s}$ for $p_{1}$ and one to two orders of magnitude smaller for the remaining components. Hence, both the linear and angular momentum can for all practical purposes be considered to be conserved. This result is anticipated, since the equations of motion have been derived from a discrete Lagrangian.

\subsection{Large-amplitude vibrations of cantilever beam}

In this example, a cantilever beam of length $6 \mathrm{~m}$ with a square cross section (side length $1 \mathrm{~m}$ ) is considered, occupying the referential domain $-0.5 \mathrm{~m} \leq X_{2}, X_{3} \leq 0.5 \mathrm{~m}$ and $0 \leq X_{1} \leq 6 \mathrm{~m}$. The beam is clamped at $X_{1}=0$ (i.e. zero displacement is prescribed) and all other boundaries are free. The initial velocity of the beam increases with increasing distance from the clamped end, according to

$\boldsymbol{v}=v_{0}\left(\begin{array}{c}0 \\ 0 \\ \frac{X_{1}}{L}\end{array}\right)$,

with $v_{0}=10 \mathrm{~m} / \mathrm{s}$, so that the beam undergoes largeamplitude oscillations in the $X_{1}-X_{3}$ plane. The material parameters are $E=17.0 \mathrm{MPa}, v=0.3$ and $\rho=$ $1.1 \times 10^{3} \mathrm{~kg} / \mathrm{m}^{3}$ and the contact parameters are set to unity $\left(\xi_{\mathrm{n}}=\xi_{\mathrm{t}}=1\right)$. Figure 8 displays the coordinates of the top right corner of the free end as a function of time for four different particle densities $(2 / \mathrm{m}, 4 / \mathrm{m}, 8 / \mathrm{m}$ and $16 / \mathrm{m})$. The period of oscillation is somewhat too small for the lowest particle density, indicating a too stiff response, but the results are similar for the remaining particle densities. The deformed shape of the beam after $0.5 \mathrm{~s}$, which corresponds the maximal deflection (see Fig. 8), is provided in Fig. 9. The results are virtually identical for particle densities $\geq 4 / \mathrm{m}$ but the deflection is somewhat smaller for the lowest particle density $(2 / \mathrm{m})$ and the stress is less uniform in this case. (a)

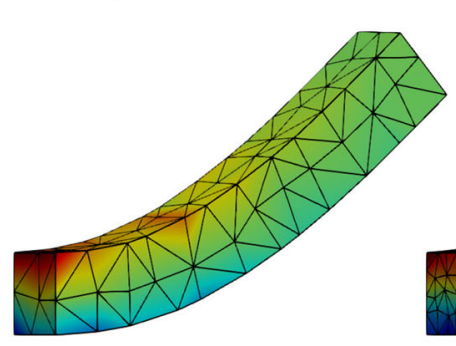

(b)

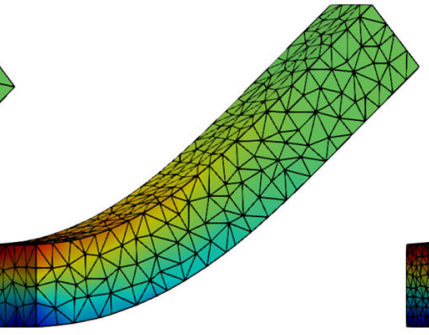

(c)

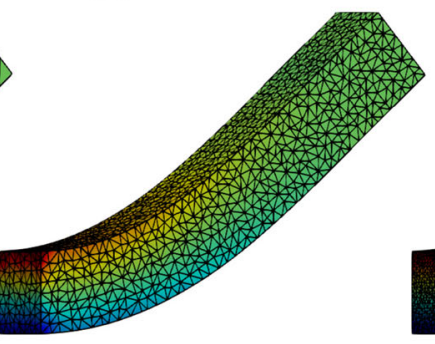

(d)

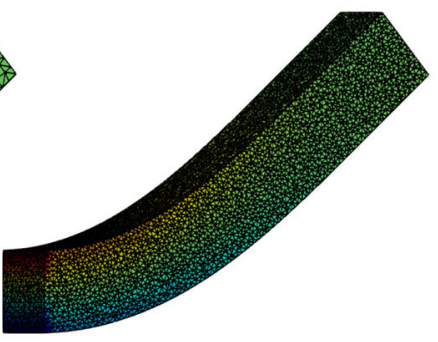

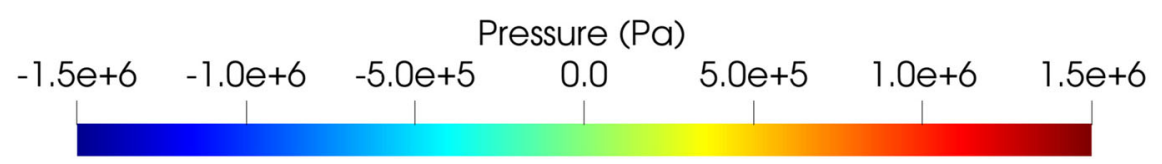

Fig. 9 Deformed shape of a cantilever beam after $0.5 \mathrm{~s}$ for four different particle densities: a $2 / \mathrm{m}, \mathbf{b} 4 / \mathrm{m}, \mathbf{c} 8 / \mathrm{m}$ and $\mathbf{d} 16 / \mathrm{m}$ (color online) 
(a)

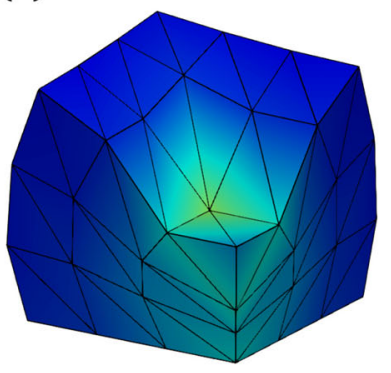

(b)

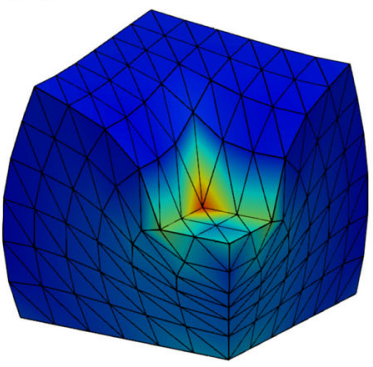

(c)

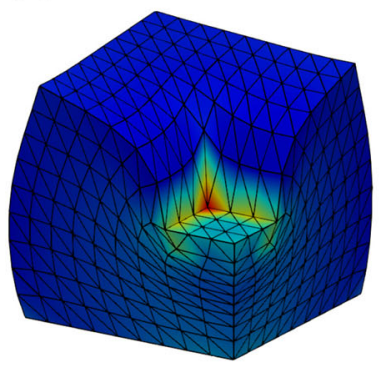

(d)

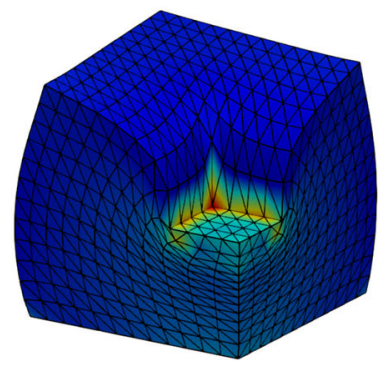

Pressure $(\mathrm{Pa})$

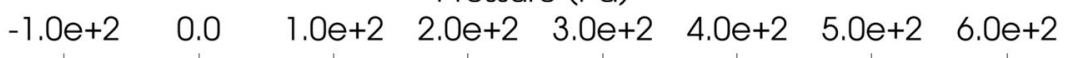

Fig. 10 Deformed shape of a rectangular block at maximum punch displacement for four different particle densities: a 3/mm, b 6/mm, c $9 / \mathrm{mm}$ and d $12 / \mathrm{mm}$ (color online)

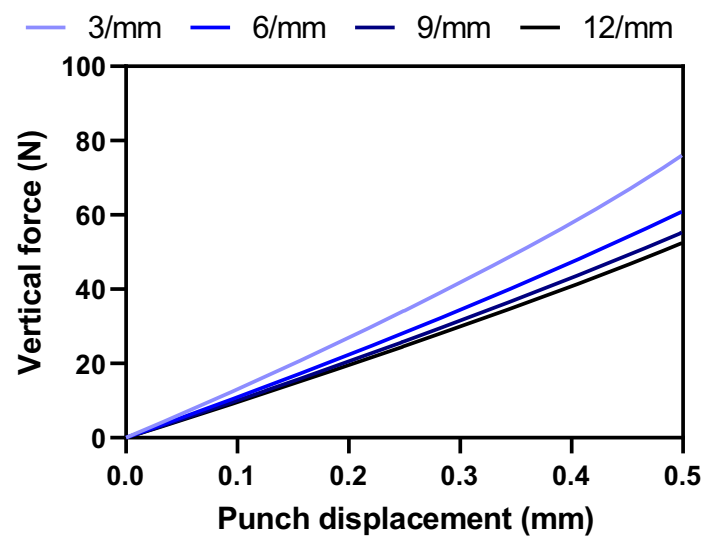

Fig. 11 Vertical force vs. punch displacement for four different particle densities

\subsection{Punch problem}

In this example, the central part of a rectangular block with dimensions $2 \times 2 \times 1 \mathrm{~mm}$ (length $\times$ width $\times$ height) is compressed by a square punch with $2 / 3 \mathrm{~mm}$ side length. Compression is done under displacement control at a rate of $0.1 \mathrm{~mm} / \mathrm{s}$ for $5 \mathrm{~s}$, resulting in a maximal punch displacement of $0.5 \mathrm{~mm}$. The bottom face of the block is kept fixed. The material parameters are $E=240 \mathrm{MPa}, v=0.4$ and $\rho=1.0 \times 10^{3} \mathrm{~kg} / \mathrm{m}^{3}$ and the contact parameters are set to unity $\left(\xi_{\mathrm{n}}=\xi_{\mathrm{t}}=1\right)$. No damping was used. Considering the symmetry of the problem, only one quarter is considered, occupying the referential domain $0 \leq X_{1}, X_{2}, X_{3} \leq 1 \mathrm{~mm}$. Zero normal displacement is enforced for $X_{1}=X_{2}=0$ (symmetric boundary conditions). A regular particle arrangement was used in order to be able to clearly see any tendency for hourglassing. The deformed shape at maximal punch displacement for four different particle densities $(3 / \mathrm{mm}, 6 / \mathrm{mm}$, 9/mm and $12 / \mathrm{mm}$ ) are displayed in Fig. 10. The overall shape of the block is similar in all cases, but the significant deformation in the vicinity of the punch boundary cannot be resolved unless a relatively high particle density is used. No tendency for hourglassing can be seen, indicating that the contact forces provided sufficient stabilisation in this case. The total vertical (punch) force is displayed as a function of punch displacement in Fig. 11. Consistent with the qualitative observations made above, the results obtained indicate that a relatively large particle density (at least $10 / \mathrm{mm}$ ) is required to obtain an adequate solution.

\section{Conclusions}

The most widely accepted Delaunay-based strain definition for granular media has been translated into a particle-based method for simulation of the mechanics of continua. The structure of the method resembles the one obtained from smoothed particle hydrodynamics or peridynamics. The determination of the deformation gradient is based on an underlying tetrahedral mesh, resulting in a formulation that is linearly consistent and satisfies the patch test. Only nearestneighbour interactions are considered, which considerably simplifies the implementation of boundaries. The interactions between particles are assumed to have two components: The first component originates from the mean stress that results from the affine (global) particle deformation (as per the standard smoothed-particle hydrodynamics). The second part emerges from non-affine (local) deformation of the contact between any two particles (in a similar manner as for the discrete element method). The particle interactions are derived from a discrete Lagrangian, thus ensuring that linear and angular momentum are conserved in the absence of external forces or torques. The performance of the method 
has been demonstrated in a number of numerical examples. This work can be considered a first step towards the development of a macroscopically consistent discrete method for granular materials.

Acknowledgements This study is part of the science program of the Swedish Drug Delivery Center (SweDeliver) and financial support from Vinnova (Dnr 2019-00048) is gratefully acknowledged. The computations were enabled by resources provided by the Swedish National Infrastructure for Computing (SNIC) at HPC2N partially funded by the Swedish Research Council through Grant Agreement No. 2018-05973.

Funding Open access funding provided by Uppsala University. Financial support was obtained from the Swedish Agency for Innovation Systems, Vinnova (Dnr 2019-00048).

Code availability Available from the author upon reasonable request.

\section{Declarations}

Conflict of interest The author declares no conflict of interest.

Open Access This article is licensed under a Creative Commons Attribution 4.0 International License, which permits use, sharing, adaptation, distribution and reproduction in any medium or format, as long as you give appropriate credit to the original author(s) and the source, provide a link to the Creative Commons licence, and indicate if changes were made. The images or other third party material in this article are included in the article's Creative Commons licence, unless indicated otherwise in a credit line to the material. If material is not included in the article's Creative Commons licence and your intended use is not permitted by statutory regulation or exceeds the permitted use, you will need to obtain permission directly from the copyright holder. To view a copy of this licence, visit http://creativecomm ons.org/licenses/by/4.0/.

\section{References}

1. Bagi K (1996) Stress and strain in granular assemblies. Mech Mater 22(3):165-177. https://doi.org/10.1016/0167-6636(95)00044-5

2. Belytschko T, Guo Y, Liu WK, Xiao SP (2000) A unified stability analysis of meshless particle methods. Int $\mathrm{J}$ Numer Methods Eng 48(9):1359-1400. https://doi.org/10.1002/10970207(20000730)48:9\%3c1359::AID-NME829\%3e3.0.CO;2-U

3. Bonet J, Gil AJ, Lee CH, Aguirre M, Ortigosa R (2015) A first order hyperbolic framework for large strain computational solid dynamics. Part I: total Lagrangian isothermal elasticity. Comput Methods Appl Mech Eng 283:689-732. https://doi.org/10.1016/j. cma.2014.09.024

4. Bonet J, Kulasegaram S, Rodriguez-Paz MX, Profit M (2004) Variational formulation for the smooth particle hydrodynamics (SPH) simulation of fluid and solid problems. Comput Methods Appl Mech Eng 193(12-14):1245-1256. https://doi.org/10.1016/j.cma. 2003.12.018

5. Bonet J, Lok TS (1999) Variational and momentum preservation aspects of smooth particle hydrodynamic formulations. Comput Methods Appl Mech Eng 180(1-2):97-115. https://doi.org/10. 1016/S0045-7825(99)00051-1

6. Celigueta MA, Latorre S, Arrufat F, Oñate E (2017) Accurate modelling of the elastic behavior of a continuum with the discrete element method. Comput Mech 60(6):997-1010. https://doi.org/ 10.1007/s00466-017-1453-9

7. Chen JS, Wu CT, Yoon S, You Y (2001) Stabilized conforming nodal integration for Galerkin mesh-free methods. Int J Numer Methods Eng 50(2):435-466. https://doi.org/10.1002/10970207(20010120)50:2\%3c435::AID-NME32\%3e3.0.CO;2-A

8. Courant R, Friedrichs K, Lewy H (1928) Über die partiellen Differenzengleichungen der mathematischen Physik. Math Ann 100:32-74

9. Cundall PA, Strack OD (1979) A discrete numerical model for granular assemblies. Geotechnique 29(1):47-65. https://doi.org/ 10.1680/geot.1979.29.1.47

10. Dohrmann CR, Heinstein MW, Jung J, Key SW, Witkowski WR (2000) Node-based uniform strain elements for three-node triangular and four-node tetrahedral meshes. Int J Numer Methods Eng 47(9):1549-1568. https://doi.org/10.1002/(SICI)10970207(20000330)47:9\%3c1549::AID-NME842\%3e3.0.CO;2-K

11. Durán O, Kruyt NP, Luding S (2010) Analysis of three-dimensional micro-mechanical strain formulations for granular materials: evaluation of accuracy. Int J Solids Struct 47(2):251-260. https://doi. org/10.1016/j.ijsolstr.2009.09.035

12. Durán O, Kruyt NP, Luding S (2010) Micro-mechanical analysis of deformation characteristics of three-dimensional granular materials. Int J Solids Struct 47(17):2234-2245. https://doi.org/10.1016/ j.ijsolstr.2010.04.014

13. Dyka CT, Ingel RP (1995) An approach for tension instability in smoothed particle hydrodynamics (SPH). Comput Struct 57(4):573-580. https://doi.org/10.1016/0045-7949(95)00059-P

14. Dyka CT, Randles PW, Ingel RP (1997) Stress points for tension instability in SPH. Int $\mathrm{J}$ Numer Methods Eng 40(13):2325-2341. https://doi.org/10.1002/(SICI)10970207(19970715)40:13\%3c2325::AID-NME161\%3e3.0.CO;2-8

15. Fraige FY, Langston PA (2004) Integration schemes and damping algorithms in distinct element models. Adv Powder Technol 15(2):227-245. https://doi.org/10.1163/156855204773644454

16. Ganzenmüller GC (2015) An hourglass control algorithm for Lagrangian smooth particle hydrodynamics. Comput Methods Appl Mech Eng 286:87-106. https://doi.org/10.1016/j.cma.2014. 12.005

17. Ganzenmüller GC, Hiermaier S, May M (2015) On the similarity of meshless discretizations of peridynamics and smoothparticle hydrodynamics. Comput Struct 150:71-78. https://doi.org/ 10.1016/j.compstruc.2014.12.011

18. Ganzenmüller GC, Sauer M, May M, Hiermaier S (2016) Hourglass control for Smooth Particle Hydrodynamics removes tensile and rank-deficiency instabilities: hourglass control for SPH. Eur Phys J Spec Top 225(2):385-395. https://doi.org/10.1140/epjst/e201602631-x

19. Giannis K, Schilde C, Finke JH, Kwade A, Celigueta MA, Taghizadeh K, Luding S (2021) Stress based multi-contact model for discrete-element simulations. Granul Matter. https://doi.org/10. 1007/s10035-020-01060-8

20. Gil AJ, Lee CH, Bonet J, Ortigosa R (2016) A first order hyperbolic framework for large strain computational solid dynamics. Part II: total Lagrangian compressible, nearly incompressible and truly incompressible elasticity. Comput Methods Appl Mech Eng 300:146-181. https://doi.org/10.1016/j.cma.2015.11.010

21. Gingold RA, Monaghan JJ (1977) Smoothed particle hydrodynamics: theory and application to non-spherical stars. Mon Not $\mathrm{R}$ Astron Soc 181(3):375-389. https://doi.org/10.1093/mnras/181.3. 375

22. Goldstein H, Poole C, Safko J (2002) Classical mechanics, 3rd edn. Pearson education, Upper Saddle River

23. Gonzalez M, Cuitiño AM (2012) A nonlocal contact formulation for confined granular systems. J Mech Phys Solids 60(2):333-350. https://doi.org/10.1016/j.jmps.2011.10.004 
24. Gray JP, Monaghan JJ, Swift RP (2001) SPH elastic dynamics. Comput Methods Appl Mech Eng 190(49-50):6641-6662. https:// doi.org/10.1016/S0045-7825(01)00254-7

25. Gurtin ME, Fried E, Anand L (2010) The mechanics and thermodynamics of continua. Cambridge University Press, Cambridge. https://doi.org/10.1017/cbo9780511762956

26. He QC (2014) On the micromechanical definition of macroscopic strain and strain-rate tensors for granular materials. Comput. Mater. Sci. 94(C):51-57. https://doi.org/10.1016/j.commatsci. 2014.01.057

27. Jonsson H, Alderborn G, Frenning G (2019) Evaluation of bulk compression using a discrete element procedure calibrated with data from triaxial compression experiments on single particles. Powder Technol 345:74-81. https://doi.org/10.1016/j. powtec.2018.12.090

28. Lee CH, Gil AJ, Ghavamian A, Bonet J (2019) A total Lagrangian upwind Smooth Particle Hydrodynamics algorithm for large strain explicit solid dynamics. Comput Methods Appl Mech Eng 344:209-250. https://doi.org/10.1016/j.cma.2018.09.033

29. Lee CH, Gil AJ, Greto G, Kulasegaram S, Bonet J (2016) A new Jameson-Schmidt-Turkel smooth particle hydrodynamics algorithm for large strain explicit fast dynamics. Comput Methods Appl Mech Eng 311:71-111. https://doi.org/10.1016/j.cma.2016. 07.033

30. Lee CH, Gil AJ, Hassan OI, Bonet J, Kulasegaram S (2017) A variationally consistent Streamline Upwind Petrov-Galerkin Smooth Particle Hydrodynamics algorithm for large strain solid dynamics. Comput Methods Appl Mech Eng 318:514-536. https://doi.org/ 10.1016/j.cma.2017.02.002

31. Libersky LD, Petschek AG (1991) Smooth particle hydrodynamics with strength of materials. In: Trease H, Fritts J, Crowley W (eds) Advanced free method, lecture notes in physics, vol 395. Springer, New York, pp 248-257. https://doi.org/10.1007/3-54054960-9_58

32. Libersky LD, Petschek AG, Carney TC, Hipp JR, Allahdadi FA (1993) High strain Lagrangian hydrodynamics a three-dimensional SPH code for dynamic material response. J Comput Phys 109(1):67-75. https://doi.org/10.1006/jcph.1993.1199

33. Littlewood DJ (2010) Simulation of dynamic fracture using peridynamics, finite element modeling, and contact. In: ASME 2020 international mechanical engineering congress and exposition, vol. 9, pp. 209-217 . https://doi.org/10.1115/IMECE2010-40621

34. Liu MB, Liu GR (2010) Smoothed particle hydrodynamics (SPH): an overview and recent developments. Arch Comput Methods Eng 17(1):25-76. https://doi.org/10.1007/s11831-010-9040-7

35. Liu MB, Liu GR, Lam KY (2003) Constructing smoothing functions in smoothed particle hydrodynamics with applications. J Comput Appl Math 155(2):263-284. https://doi.org/10.1016/ S0377-0427(02)00869-5

36. Lucy LB (1977) A numerical approach to the testing of the fission hypothesis. Astron J 82:1013. https://doi.org/10.1086/112164

37. Mattsson AE, Rider WJ (2015) Artificial viscosity: back to the basics. Int J Numer Methods Fluids 77(7):400-417. https://doi. org/10.1002/fld.3981

38. Monaghan JJ (1985) Particle methods for hydrodynamics. Comput Phys Rep 3(2):71-124. https://doi.org/10.1016/01677977(85)90010-3

39. Monaghan JJ, Gingold RA (1983) Shock simulation by the particle method SPH. J Comput Phys 52(2):374-389. https://doi.org/10. 1016/0021-9991(83)90036-0

40. Monaghan JJ, Pongracic H (1985) Artificial viscosity for particle methods. Appl Numer Math 1(3):187-194. https://doi.org/10. 1016/0168-9274(85)90015-7

41. Pelupessy FI, Schaap WE, Van de Weygaert R (2003) Density estimators in particle hydrodynamics. DTFE versus regular SPH.
Astron Astrophys 403(2):389-398. https://doi.org/10.1051/00046361:20030314

42. Puso MA, Chen JS, Zywicz E, Elmer W (2008) Meshfree and finite element nodal integration methods. Int J Numer Methods Eng 74(3):416-446. https://doi.org/10.1002/nme.2181

43. Rabczuk T, Belytschko T, Xiao SP (2004) Stable particle methods based on Lagrangian kernels. Comput Methods Appl Mech Eng 193(12-14):1035-1063. https://doi.org/10.1016/j.cma.2003. 12.005

44. Rausch MK, Karniadakis GE, Humphrey JD (2017) Modeling soft tissue damage and failure using a combined particle/continuum approach. Biomech Model Mechanobiol 16(1):249-261. https:// doi.org/10.1007/s10237-016-0814-1

45. Schaap WE, Van De Weygaert R (2000) Continuous fields and discrete samples: Reconstruction through Delaunay tessellations. Astron Astrophys 363(3)

46. Scovazzi G, Carnes B, Zeng X, Rossi S (2016) A simple, stable, and accurate linear tetrahedral finite element for transient, nearly, and fully incompressible solid dynamics: a dynamic variational multiscale approach. Int J Numer Methods Eng 106(10):799-839. https://doi.org/10.1002/nme.5138

47. Si H (2015) TetGen, a Delaunay-based quality tetrahedral mesh generator. ACM Trans Math Softw. https://doi.org/10.1145/ 2629697

48. Silling SA (2000) Reformulation of elasticity theory for discontinuities and long-range forces. J Mech Phys Solids 48(1):175-209. https://doi.org/10.1016/S0022-5096(99)00029-0

49. Silling SA (2017) Stability of peridynamic correspondence material models and their particle discretizations. Comput Methods Appl Mech Eng 322:42-57. https://doi.org/10.1016/j.cma.2017. 03.043

50. Silling SA, Epton M, Weckner O, Xu J, Askari E (2007) Peridynamic states and constitutive modeling. J Elast 88(2):151-184. https://doi.org/10.1007/s10659-007-9125-1

51. Silling SA, Lehoucq RB (2010) Peridynamic theory of solid mechanics. In: Aref H, van der Giessen E (eds) Advances in applied mechanics, vol 44. Elsevier, Amsterdam, pp 73-168. https://doi. org/10.1016/S0065-2156(10)44002-8

52. Swegle JW, Hicks DL, Attaway SW (1995) Smoothed particle hydrodynamics stability analysis. J Comput Phys 116(1):123-134. https://doi.org/10.1006/jcph.1995.1010

53. Taylor RL, Simo JC, Zienkiewicz OC, Chan AC (1986) The patch test-a condition for assessing FEM convergence. Int J Numer Methods Eng 22(1):39-62. https://doi.org/10.1002/nme.1620220105

54. Vidal Y, Bonet J, Huerta A (2007) Stabilized updated Lagrangian corrected SPH for explicit dynamic problems. Int J Numer Methods Eng 69(13):2687-2710. https://doi.org/10.1002/nme.1859

55. Zeng W, Liu GR, Jiang C, Nguyen-Thoi T, Jiang Y (2016) A generalized beta finite element method with coupled smoothing techniques for solid mechanics. Eng Anal Bound Elem 73:103119. https://doi.org/10.1016/j.enganabound.2016.09.008

56. Zhang A, Ming F, Cao X (2014) Total Lagrangian particle method for the large-deformation analyses of solids and curved shells. Acta Mech 225(1):253-275. https://doi.org/10.1007/s00707-0130938-7

Publisher's Note Springer Nature remains neutral with regard to jurisdictional claims in published maps and institutional affiliations. 